\title{
Características mecánicas y térmicas de un poliuretano elaborado a partir de aceite de higuerilla (ricinus communis) para la adhesión de elementos estructurales de guadua angustifolia kunth Mechanical and thermal characteristics of a polyurethane processed from hyguerilla oil (ricinus communis) for the adhesion of structural elements of guadua angustifolia kunth
}

\author{
Fernando Gordillo-Delgado ${ }^{1 a, 2}$, Adrián Felipe Bedoya-Pérez ${ }^{1 b, 2}$, Hector David Delgado- \\ Osorio $^{3}$
}

\footnotetext{
${ }^{1}$ Grupo de Investigación en Ciencia Aplicada para el Desarrollo de la Ecorregión (GICADE), Universidad del Quindío, Colombia. Correos electrónicos: fgordillo@uniquindio.edu.co, labopt@uniquindio.edu.co

${ }^{2}$ Instituto Politécnico Nacional, México.

${ }^{3}$ Programa de Tecnología en Instrumentación Electrónica de la Facultad de Ciencias Básicas y Tecnologías, Universidad del Quindío, Colombia. Correo electrónico: fotoacustica@uniquindio.edu.co
}

Recibido: 15 diciembre, 2019. Aceptado: 30 abril, 2020. Versión final: 24 julio, 2020.

\begin{abstract}
Resumen
En las últimas décadas se ha intensificado la búsqueda de materia prima proveniente de recursos renovables para la producción de polímeros, elastómeros, resinas, espumas, etc., que disminuyan el uso industrial de compuestos petroquímicos. En este trabajo se usó el aceite extraído de la semilla de higuerilla (Ricinus communis) por su contenido de ácido ricinoleico $(92,3 \%)$, por su fácil producción y por no ser comestible; la síntesis del poliuretano adhesivo se hizo a través de reacciones de transesterificación y polimerización; con él se pegaron láminas de Guadua angustifolia Kunth y se determinaron las características mecánicas de la estructura; se obtuvo que la resistencia a la compresión

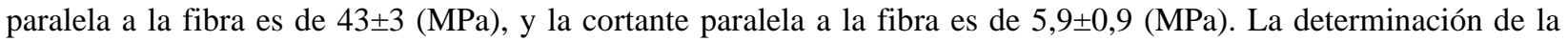
relación molar adecuada y el seguimiento de la reacción durante la síntesis se hicieron con espectroscopía infrarroja con transformada de Fourier y la estructura del producto final se analizó con difracción de rayos X. A través de calorimetría diferencial de barrido se obtuvo la temperatura de transición vítrea del producto de $-26.4{ }^{\circ} \mathrm{C}$ y se usó la técnica de relajación térmica para medir su calor específico, mientras que su difusividad térmica y el coeficiente de dilatación térmica se estimaron con la técnica fotoacústica.
\end{abstract}

Palabras clave: adhesivo; aceite de ricino; difusividad térmica; calor específico; fotoacústica; relajación térmica; resistencia mecánica.

ISSN impreso: 1657 - 4583. ISSN en línea: 2145 - 8456, CC BY-ND 4.0 다 (1) 요

Como citar: J. F. Gordillo-Delgado, A. F. Bedoya-Pérez, H. D. Delgado-Osorio, "Características mecánicas y térmicas de un poliuretano elaborado a partir de aceite de higuerilla (ricinus communis) para la adhesión de elementos estructurales de guadua angustifolia kunth," Rev. UIS Ing., vol. 19, no. 4, pp. 103-114, 2020, doi: https://doi.org/10.18273/revuin.v19n4-2020009 


\begin{abstract}
In recent decades, the search for raw from renewable resources to produce polymers, elastomers, resins, foams, etc., has intensified; these materials would reduce the industrial use of petrochemical compounds. In this work, the oil extracted from the castor oil seed (Ricinus communis) was used because of ricinoleic acid content (92.3\%), it is of easy production and non-edible vegetable origin. The polyurethane adhesive was synthesized through transesterification and polymerization reactions. Using the corresponding standards, this adhesive was applied to sheets of Guadua angustifolia Kunth for determining its mechanical characteristics, the compressive strength parallel to the fiber was $43 \pm 3(\mathrm{MPa})$, and shear parallel to the fiber was $5.9 \pm 0.9(\mathrm{MPa})$. Fourier transform infrared spectroscopy was used for determining the appropriate molar ratio and monitoring the reaction during the synthesis and final product structure was analyzed with X-ray diffraction. Through differential scanning calorimetry, the glass transition temperature of the product was estimated on $-24.6{ }^{\circ} \mathrm{C}$; the thermal relaxation technique was used to measure its specific heat, while its thermal diffusivity and thermal expansion coefficient were estimated with the photoacoustic technique.
\end{abstract}

Keywords: adhesive; castor oil; thermal diffusivity; specific heat; photoacoustic; thermal relaxation; mechanical resistance.

\section{Introducción}

Los adhesivos son un insumo básico en la industria maderera dedicada a la fabricación de muebles, de materiales de construcción o de elementos decorativos. Los fabricantes deben garantizar la estabilidad de la estructura frente a factores como la humedad, la temperatura y los esfuerzos mecánicos. Por esta razón, los pegamentos deben tener características adecuadas para cada uso y tipo de madera. Entre los adhesivos sintéticos con mayor eficacia se encuentra el fenol ureaformaldehido, que presenta desventajas como producción con alto consumo de energía, toxicidad y precio de su principal componente, el fenol.

La elaboración de adhesivos se basa en el uso de las propiedades de los polímeros [1]; los polisacáridos y las proteínas son polímeros naturales de alto peso molecular, derivados de plantas y animales; la piel, la sangre, la caseína, el almidón, la soja, la dextrina, y la celulosa son algunas de las fuentes principales [2]. Estas fueron utilizadas durante mucho tiempo en la fabricación de pegantes y aunque hoy en día son de uso doméstico, se reemplazaron a escala industrial por los sintéticos, que son efectivos [3], pero con alto costo ambiental de producción.

Con el propósito de mejorar las características fisicoquímicas y la funcionalidad de los poliuretanos, algunos trabajos se han encaminado al reemplazo de materias primas tradicionales por otras de origen vegetal, que lleven a productos y procesos económicamente viables e introduzcan propiedades de biodegradabilidad $[4,5]$. En particular, la síntesis de elastómeros de poliuretano con aceite de higuerilla (Ricinus commnunis) permite obtener un amplio rango de propiedades físicas y mecánicas, dependiendo de los contenidos de aceite y de diisocianato reaccionante. La composición y la relación de grupos funcionales $\mathrm{NCO} / \mathrm{OH}$ de los segmentos del poliuretano afectan la estructura, organización y flexibilidad del polímero y por consiguiente su morfología y propiedades mecánicas [67]. Los poliuretanos obtenidos a partir del aceite de ricino son típicamente flexibles, debido a las largas cadenas de ácidos grasos en su estructura y se caracterizan por ser resistentes a la abrasión. La estructura de estos elastómeros es termoestable debido a la naturaleza trihidroxílica del aceite. Sin embargo, algunos productos presentan desventajas como baja resistencia al rasgado, a la oxidación y al calor [8].

En las últimas décadas se han usado principalmente dos métodos para aumentar la cantidad de grupos hidroxilos (-HO) de este aceite vegetal; uno se basa en la adición de compuestos con alto contenido de este grupo funcional, como almidón, celulosa y lignina, obtenidos a partir de biomasa vegetal (fibras de coco, caña de azúcar, cáscara de arroz, cáscara de piña, pergamino de café, tusa de maíz, etc.) $[9,10]$ y el otro emplea la transesterificación del aceite con alcoholes polihidroxílicos como la glicerina y el pentaerytrol para aumentar el grado de entrecruzamiento del poliuretano [11].

El proceso de transesterificación pertenece a una clase de reacciones orgánicas en las que un éster es transformado en otro a través del intercambio del grupo alcoxi. Cuando éste se aplica a aceites vegetales, un triglicérido reacciona con un alcohol en presencia de un ácido o base fuerte; esto produce una mezcla de ésteres alquílicos de ácidos grasos y glicerol. El proceso total es una secuencia de tres reacciones consecutivas irreversibles en las que se forman mono y diglicéridos, como productos intermedios [12]. La aplicación de este método conduce al mejoramiento de las propiedades tensiles, dureza y resistencia al ataque químico, debido al mayor grado de entrecruzamiento ocasionado por el incremento del contenido de grupos hidroxilo [13]. 
En la elaboración de elementos estructurales se emplean adhesivos para la unión de fibras o de tablillas; de tal forma que un buen encolado es fundamental para obtener características mecánicas adecuadas. La guadua (Guadua angustifolia-Kunth) es un recurso forestal importante [14], ya que es una planta de rápido crecimiento que puede ser un buen sustituto de la madera bajo estrategias de reforestación y producción razonables. El diseño y la fabricación de elementos estructurales como laminados, paneles, tableros, cerchas o vigas prefabricadas con guadua se ha convertido en un tema de gran interés en los últimos años y en el mercado internacional ya existe una oportunidad de comercio viable [15]. El crecimiento del mercado de ecomateriales, que son premiados con un sobreprecio [16], así como el interés por mitigar los efectos ambientales de la tala de árboles en la industria forestal y por disminuir la utilización de recursos no renovables, hacen que sea necesario el desarrollo y la evaluación de pegantes de origen natural.

El fenol/formaldehido (PF), la urea/formaldehido (UF) y los isocianatos (MDI) son las resinas inorgánicas derivadas del petróleo más utilizadas en la industria maderera. La primera es sensible a la humedad, pero presenta alta resistencia al calor; mientras que las dos últimas son aptas para uso en exteriores y revestimientos superficiales. Sin embargo, tanto el formaldehido, como los isocianatos y el fenol son tóxicos [17]. Por esta razón, es oportuno el aprovechamiento de productos naturales para la fabricación de adhesivos orgánicos, con los que se obtengan características físicas similares a las que se consiguen con encolados sintéticos [18,]; en particular, varios autores han encontrado materiales con comportamiento mecánico adecuado, utilizando pegantes hechos con ricino [22].

En este trabajo, se caracterizaron térmica y mecánicamente elementos estructurales de Guadua angustifolia Kunth, fabricados con un adhesivo preparado con aceite vegetal, extraído de la semilla de la higuerilla. A través de la caracterización, tanto del pegante como de los elementos laminados, se evaluaron los parámetros usados en la síntesis [19-20] y se usó la proporción de los materiales mezclados con la más alta componente de la fuente natural usada. Se obtuvieron espectros de absorción del producto por medio de espectroscopia para determinar la composición y la funcionalidad del aceite en el proceso de transesterificación.

Los respectivos valores de los parámetros termofísicos y del coeficiente de dilatación térmica del adhesivo se estimaron por medio de una técnica fototérmica y otra de relajación térmica y se usó la calorimetría diferencial de barrido para probar su estabilidad frente a cambios de temperatura [21]. La eficiencia del producto a solicitaciones requeridas por las normas de calidad se comprobó a través de pruebas mecánicas sobre elementos estructurales hechos con guadua, de las que se obtuvo la resistencia de las uniones encoladas. Los resultados muestran el potencial del pegante para suplir una necesidad de la emergente industria de la guadua como ecomaterial. Por otro lado, las mediciones que se hicieron en este trabajo validan la aplicación de las técnicas empleadas en la caracterización térmica de materiales de origen biológico.

\section{Modelado de la red eléctrica}

Las muestras de guadua, previamente curadas y secadas, se compraron en Armenia (Departamento del Quindío) y se dividieron en varillones de $1.7 \mathrm{~m}$ desde el rizoma hasta el tramo conocido como basa. Estos se cortaron en latas, utilizando cuchillas radiales, y se secaron al aire libre hasta alcanzar un porcentaje de humedad entre 10 y 12 $\%$. Luego de quitar los nudos y los imperfectos se procedió a ajustar el ancho y el espesor de las latas extraídas, por medio de cuchillas paralelas. Las probetas se armaron con aplicación del adhesivo y secado bajo presión durante 24 horas, a temperatura ambiente. Los tableros de fibra compactada se hicieron con una mezcla de aserrín de guadua y pegamento, prensada a una temperatura de $50{ }^{\circ} \mathrm{C}$ durante 1 hora.

El proceso para la extracción del aceite de ricino se inició con la recolección de los frutos oleaginosos de higuerilla; estos se colocaron en un horno de convección durante 6 horas, a una temperatura de $110{ }^{\circ} \mathrm{C}$, para disminuir su contenido de humedad a menos del $10 \%$. En la siguiente etapa, se separó mecánicamente la cáscara de la semilla y luego se molieron estos granos con un molino (extrusor PITEBA); el residuo de cáscara rígida y almendra se llevaron a un sistema Soxhlet durante 4 horas a una temperatura de $70{ }^{\circ} \mathrm{C}$, utilizando un solvente orgánico (hexano con pureza del $99.7 \%$ ); de esta forma se extrajo el $20 \%$ del aceite residual, contenido en la torta compactada y se recuperó el solvente mediante técnicas de destilación y evaporación. Por último, se calentaron los dos productos obtenidos para disminuir la viscosidad y se pasaron por un filtro para la remoción de sólidos; la neutralización o disminución de la acidez del aceite se hizo con $\mathrm{NaOH}$.

El adhesivo de origen natural se elaboró con este aceite; el $90 \%$ de su contenido corresponde al ácido ricinoléico en forma de triglicérido y es uno de los pocos glicéridos naturales casi puros. El ácido triglicérido fue hidroxilado por transesterificación, rompiendo su estructura de anillo; luego, el poliol y un isocianato (MDI) se llevaron 
a un reactor en presencia de un catalizador en medio acuoso. En esta etapa ocurrieron dos reacciones simultáneas: el isocianato reaccionó con el poliol para formar poliuretano y con el agua para generar poliurea y dióxido de carbono (que actúa como agente espumante). Finalmente, el producto fue enfriado durante 48 horas.

El desarrollo de las pruebas mecánicas se hizo de acuerdo con las normas de la ASTM (American Society for Testing and Materials) usando una máquina universal de ensayos Ibertest (UMIB-600-SW). Se analizó la estabilidad térmica del pegante, utilizando la técnica fotoacústica (FA) y calorimetría por relajación térmica. La FA en configuración de variación de frecuencia se usó para estimar los valores de difusividad térmica y el coeficiente de expansión térmica; en el montaje experimental se usó un láser de argón que emite un haz de luz de longitud de onda de $488 \mathrm{~nm}$, que se corta periódicamente mediante un modulador acusto-óptico. El haz modulado se hace incidir sobre la muestra, colocada en una celda que tiene integrado un micrófono de electret. Para obtener los espectros de absorción en el infrarrojo medio (entre $400 \mathrm{~cm}-1$ y $4000 \mathrm{~cm}-1$ ) se empleó un espectrofotómetro SHIMADZU Prestige 21. Las muestras del adhesivo y las latas de guadua se almacenaron a una temperatura de $20{ }^{\circ} \mathrm{C}$ y a una humedad de $60 \%$, en un climatizador. Las pruebas de ataque químico se hicieron por inmersión durante diez días en varios agentes, de acuerdo con la norma ASTM D543-14.

La elaboración del pegante se hizo bajo condiciones de laboratorio, garantizando el cuidado y manejo adecuado de almacenamiento y uso de los reactivos y de los desechos. El proceso de transesterificación descrito no implica la emisión de gases contaminantes y durante la aplicación y secado no se generaron gases tóxicos, dadas las características del adhesivo y del método de fabricación de los elementos estructurales por compresión a temperatura ambiente. La síntesis de este pegamento de origen natural es amigable con el medio ambiente y las estructuras de guadua, que con él se fabricaron, tienen características de resistencia mecánica que las convierten en una alternativa para el uso de la guadua y de los desechos que se generan de su procesado para obtener tablillas.

\section{Resultados}

\subsection{Fabricación del pegamento}

silvestres, dentro del campus de la Universidad del Quindío; se les hizo un proceso de secado y descascarado. La extracción química del aceite se hizo mediante un proceso de reflujo Soxhlet, partiendo de las semillas previamente molidas $y$ se obtuvo un rendimiento de $43 \%$, usando hexano. En la tabla 1 se hizo el análisis del espectro FTIR del aceite, que se muestra en la figura 1.

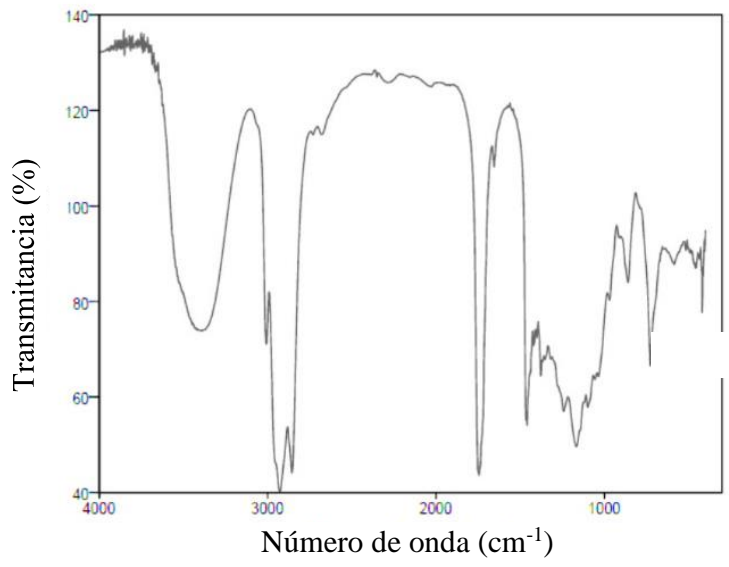

Figura 1. Espectro FTIR del aceite de higuerilla. Fuente: elaboración propia.

Tabla 1. Análisis del espectro FTIR que se muestra en la figura 1 , correspondiente al aceite de higuerilla

\begin{tabular}{|c|c|}
\hline $\begin{array}{l}\text { Número de } \\
\text { onda }\left(\mathrm{cm}^{-1}\right)\end{array}$ & Grupo Químico \\
\hline$<1500$ & $\begin{array}{l}\text { Huella digital del triglicérido del } \\
\text { ácido ricinoleico. }\end{array}$ \\
\hline 1750 & $\begin{array}{l}\text { Se confirma la presencia de } \\
\text { carbonilo; bandas entre } 1540 \mathrm{~cm}^{-1} \mathrm{y} \\
1850 \mathrm{~cm}^{-1} \text {; grupo carbonilo del } \\
\text { grupo éster entre } 1750 \mathrm{~cm}^{-1} \text { y } 1715 \\
\mathrm{~cm}^{-1} \text { (color rojo en el esquema de la } \\
\text { figura 2). }\end{array}$ \\
\hline $\begin{array}{c}2856-1467- \\
1378-1369- \\
726\end{array}$ & $\begin{array}{l}\text { Presencia de } 3 \text { hombros entre } 2800 \\
\text { y } 3000 \mathrm{~cm}^{-1} \text {, correspondientes a } \\
\text { grupos } \mathrm{CH}_{2} \text { y } \mathrm{CH}_{3} \text {. Se confirma la } \\
\text { representación de estos grupos por } \\
\text { las bandas entre } 1475 \mathrm{~cm}^{-1} \text { y } 1445 \\
\mathrm{~cm}^{-1} \text { y entre } 1385 \mathrm{~cm}^{-1} \text { y } 1365 \mathrm{~cm}^{-1} \text {; } \\
\text { mientras que la de grupos } \mathrm{CH}_{2} \text {, } \\
\text { dentro de una cadena mayor de } 4 \\
\text { carbonos, por las bandas entre } 730 \\
\mathrm{~cm}^{-1} \text { y } 710 \mathrm{~cm}^{-1} \text { (color verde en el } \\
\text { esquema de la figura } 2 \text { ). }\end{array}$ \\
\hline $3406-1099$ & $\begin{array}{l}\text { El grupo hidroxilo, asociado con un } \\
\text { puente de hidrógeno, está } \\
\text { relacionado con los hombros } \\
\text { ubicados en } 3400 \text { y } 3200 \mathrm{~cm}^{-1} \text {. Se } \\
\text { confirma el grupo de carbono } \\
\text { secundario, representado por la } \\
\text { banda alrededor de } 1100 \mathrm{~cm}^{-1} \text { (color } \\
\text { azul en el esquema de la figura } 2 \text { ). }\end{array}$ \\
\hline
\end{tabular}

Fuente: elaboración propia. 
De acuerdo con la norma NTC 218:2011 se determinó el índice de acidez del aceite, con un valor de $2.17 \mathrm{mg}$ $\mathrm{KOH} / \mathrm{g}$ (muestra); mientras que el índice de hidroxilo, de 179.3, se obtuvo siguiendo la norma NTC 1529:1998, vigente para "aceite puro de castor (aceite de ricino) para la industria de cosméticos", a través de una mezcla de anhídrido acético y piridina.

En el proceso de elaboración del pegante se modificó el poliol (60\% del peso total) por adición de diisocianato de difenilmetano (MDI) para obtener el adhesivo de poliuretano. En la figura 3 se puede ver la influencia que tiene la proporción utilizada de MDI, a través de la modificación en el espectro FTIR; se observa el efecto causado por una cantidad excesiva de este compuesto en comparación con la relación $\mathrm{NCO} / \mathrm{OH}$ de equilibrio (Figura 3 a).

a)

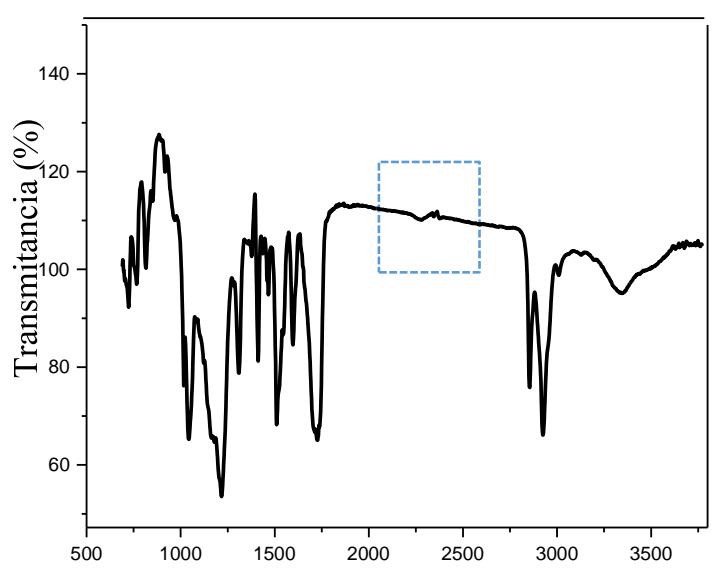

b)

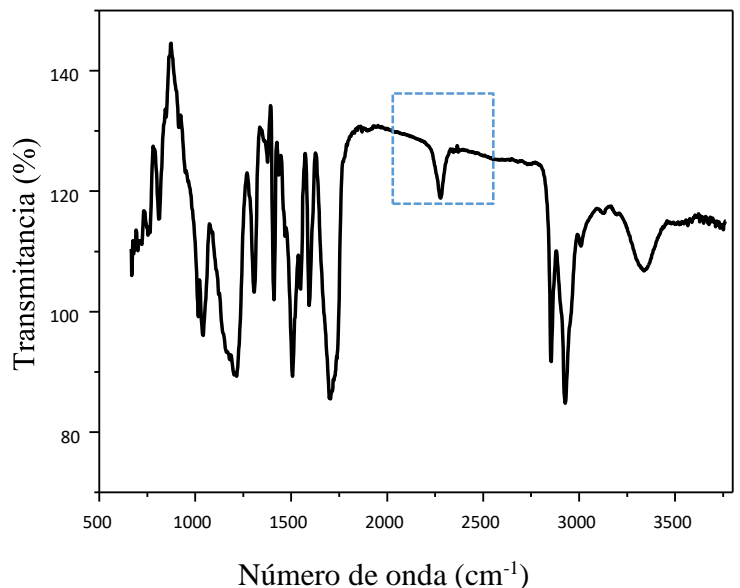

Figura 3. Espectro FTIR del adhesivo a) con la relación $\mathrm{NCO} / \mathrm{OH}$ de equilibrio y b) con exceso de NCO. Fuente: elaboración propia.
Como se puede ver en las figuras 3 a) y 3 b), entre 2100 y $2250 \mathrm{~cm}^{-1}$, no se observa la banda correspondiente al grupo del isocianato en el espectro del producto obtenido con la relación de equilibrio. Lo que indica una reacción completa para la formación del poliuretano; es decir, después de la síntesis no quedan moléculas libres de este compuesto. La banda entre 3300 y $3500 \mathrm{~cm}^{-1}$ corresponde al estiramiento de los grupos $\mathrm{N}-\mathrm{H}$, mientras que los picos entre 1640 y $1730 \mathrm{~cm}^{-1}$ se asocian a modos de vibración del grupo carbonilo, presente en los uretanos. Por otro lado, la banda pronunciada entre 2800 y $3000 \mathrm{~cm}^{-1}$ hace referencia a los estiramientos de enlace de los grupos C-H y los que aparecen entre 1480 y 1640 $\mathrm{cm}^{-1}$, a la interacción C-N.

\subsection{Caracterización del pegante}

En la tabla 2 se encuentra el promedio de la pérdida de peso de las muestras del adhesivo sólido, en la prueba de resistencia a algunos reactivos químicos. Se observaron cambios leves del peso del pegante, que conserva su apariencia para la mayoría de los productos utilizados; en particular la reacción al agua, con la que puede haber mayor contacto en aplicaciones prácticas, dio lugar a un desgaste de apenas $0.2 \%$. Se observó abultamiento con el ácido sulfúrico y acetona y deformación únicamente con éter-etílico.

Tabla 2. Cambio físico del adhesivo por inmersión en reactivos químicos durante diez días

\begin{tabular}{|l|c|}
\hline \multicolumn{1}{|c|}{ Reactivo } & Pérdida de peso (\%) \\
\hline $\mathrm{HCl}(0.1 \mathrm{~N})$ & 2.3 \\
\hline $\mathrm{H}_{2} \mathrm{SO}_{4}(0.1 \mathrm{~N})$ & 0.6 \\
\hline Éter-etílico & 6.1 \\
\hline Etanol $(95 \%)$ & 0.8 \\
\hline Hexano & 3.0 \\
\hline Metanol & 9.6 \\
\hline Acetona & 0.7 \\
\hline $\mathrm{NaOH}(0.1 \mathrm{~N})$ & 7.7 \\
\hline $\mathrm{KOH}(0.1 \mathrm{~N})$ & 2.4 \\
\hline $\mathrm{H}_{2} \mathrm{O}$ & 0.2 \\
\hline
\end{tabular}

Fuente: elaboración propia.

En el termograma de la figura 4 se observa una caída de la curva en $-26.4{ }^{\circ} \mathrm{C}$, que indica el cambio del flujo de calor, relacionado con un aumento en la capacidad calorífica. Esta temperatura de transición vítrea (Tg) es el valor por encima del cual no se quiebra la muestra. Este dato es el mismo del policloruro de vinilildeno y aunque para algunos materiales como polietileno PEAD y PEBD 
este valor está en un rango más amplio, entre -35 y -120 ${ }^{\circ} \mathrm{C}$ [22], la Tg hallada es suficiente para que el pegante conserve sus características en un rango amplio de condiciones climáticas.

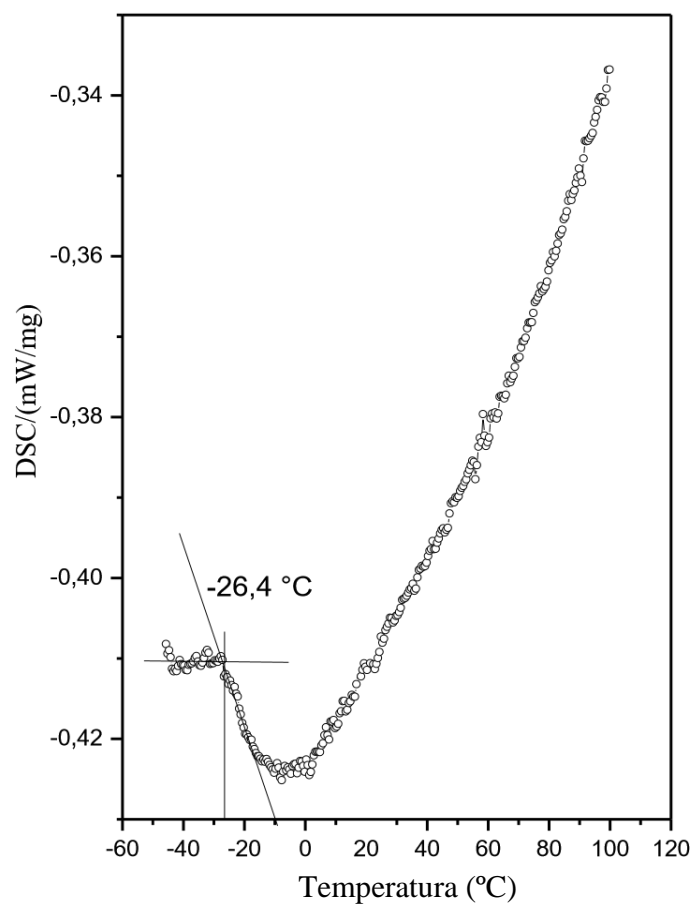

Figura 4. Termograma del adhesivo sintetizado. Fuente: elaboración propia.

Se utilizó la difracción de rayos X para determinar la estructura del producto y obtener información sobre la cristalinidad del poliuretano. En el difractograma de la figura 5 se observa un pico en $2 \Theta=17^{\circ}$, relacionado con la cristalización de las partes blandas del elastómero.

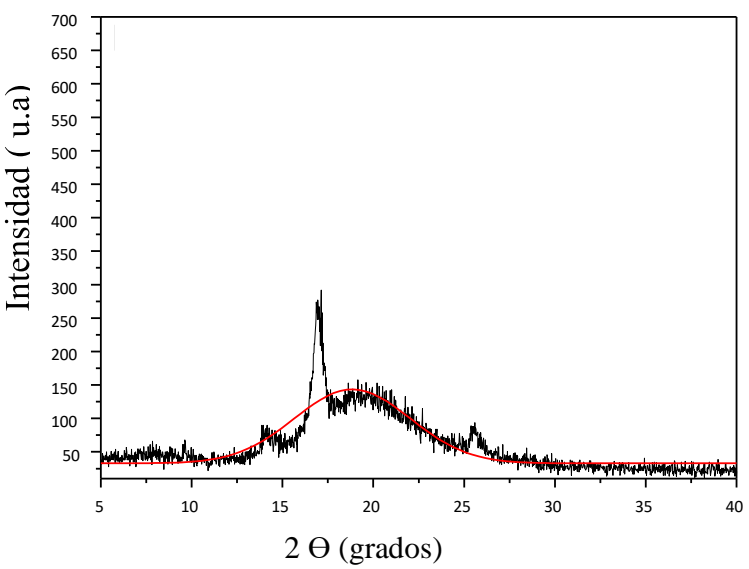

Figura 5. Difractograma de rayos X del adhesivo. Fuente: elaboración propia.
El pico ancho que va desde $12.5^{\circ}$ a $25^{\circ}$ justifica la naturaleza amorfa del adhesivo. Esta característica es similar a la que presentan las resinas epóxicas y se debe a la dispersión por las moléculas del pegante, que se forman luego del proceso de curado [23].

\subsection{Pruebas mecánicas de probetas (elementos laminados) hechas con el pegante}

A continuación, se muestran los resultados de las pruebas mecánicas, aplicadas a las probetas representadas en la figura 6, que se hicieron de acuerdo con los estándares de la ASTM; específicamente se siguió la norma ASTM D143: "Métodos de ensayo estándar para muestras pequeñas de madera".

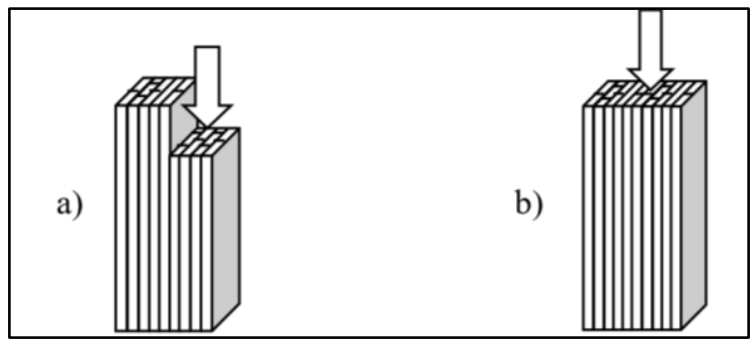

Figura 6. Determinación de a) la resistencia al cizallamiento paralelo a la fibra y de b) la compresión paralela a la fibra. Fuente: elaboración propia.

Doce probetas de guadua se sometieron a la prueba cortante paralela a la fibra y se obtuvo una resistencia de $5.9 \pm 0.9 \mathrm{MPa}$, con un coeficiente de variación de 15.01 $\%$. Este valor es similar al reportado por algunos autores para elementos de guadua, entre 4.7 y $9.4 \mathrm{MPa}$ [24]. El ensayo mecánico termina cuando la máquina detecta un movimiento abrupto llamado falla. Esto puede ser generado por fisuras en dirección horizontal, aplastamiento total, corte a lo largo de las fibras y falla de adhesión en las probetas. En este caso, la falla se presentó en las fibras de las tablillas de guadua adheridas con el pegante de higuerilla.

De acuerdo con la misma norma, se hicieron las pruebas de compresión paralela a la fibra y se obtuvo una resistencia de las probetas de $43 \pm 3 \mathrm{MPa}$, con un coeficiente de variación de $6.83 \%$. En este caso, el $90 \%$ de las muestras sufrieron falla de fisura horizontal. Este esfuerzo que soporta el material puede llegar a ser hasta 10 veces mayor que el correspondiente a ensayos perpendiculares a la fibra, lo que indica alta resistencia a la deformación estructural [16]. El valor medido es $30 \%$ más bajo que el reportado por Correal et al [25] para laminados de este mismo tipo de guadua adheridos con una mezcla de urea-formaldehído $(50 \%)$ y melanina- 
formaldehído (50\%), de $62 \mathrm{MPa}$, con un coeficiente de variación de $3.1 \%$.

Las pruebas de adherencia del pegante sobre latas de guadua se hicieron a través de un proceso como el que se muestra en la figura 7, en el que se esparció el adhesivo sobre la superficie de la probeta y se dejó secar; posteriormente, sobre la capa seca de adhesivo se pegó el cabezal de prueba, llamado "Dolly" de $20 \mathrm{~mm}$ de diámetro, por prensado durante un tiempo de 48 horas con un pegante époxico flexible (Resinalab EP11HT Gray) que tiene una fuerza de tensión que supera el límite máximo de rendimiento del sistema de presión en condiciones ideales. Finalmente, con un equipo Positest AT-M, de 0 a $0.345 \mathrm{MPa} / \mathrm{s}$ se aplicó presión y se obtuvo falla cohesiva (desprendimiento de sustrato) cuando la presión llegó, en promedio, a 1.0 $\pm 0.2 \mathrm{MPa}$; mientras que con un pegante convencional para madera (marca carpincol) se obtuvo un valor de $0.9 \pm 0.2 \mathrm{MPa}$.

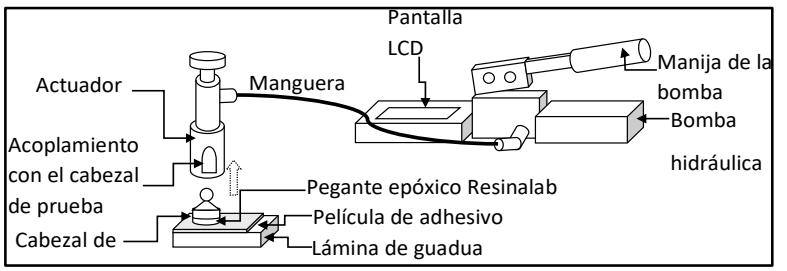

Figura 7. Imágenes del sistema usado para la medición de la adherencia del pegante a una superficie de guadua, usando un cabezal de prueba (Dolly), con un equipo

Positest AT-M. Fuente: elaboración propia.

\subsection{Caracterización térmica del adhesivo}

\subsubsection{Medición del calor específico del adhesivo}

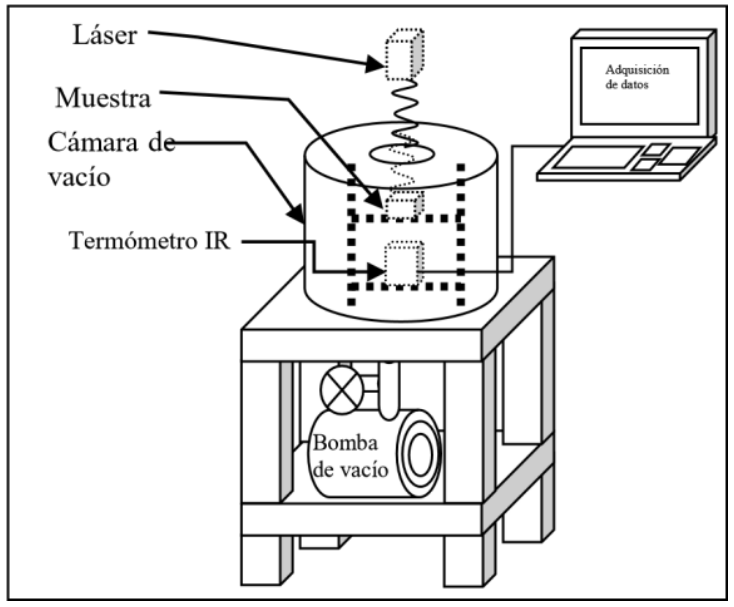

Figura 8. Esquema del sistema de RT para medición de calor específico. Fuente: elaboración propia.
En la figura 8 se muestra un esquema del sistema experimental de relajación térmica (RT) que se usó para medir el calor específico del adhesivo. La muestra se radia con un haz de luz láser (en este caso de longitud de onda de $480 \mathrm{~nm}$ ) y se registran las variaciones de temperatura, utilizando termometría IR; esta medición se repite dentro de una cámara cilíndrica de acero inoxidable de $400 \mathrm{~mm}$ de alto y $250 \mathrm{~mm}$ de diámetro, en la que se hace vacío mediante una bomba Leybold Trivac D 2,5 E, con la que se alcanza una presión interna de alrededor de $2 \times 10^{-3}$ mbar. En su configuración convencional, esta técnica se basa en la perturbación, mediante iluminación constante, del estado de equilibrio termodinámico de una muestra suspendida adiabáticamente dentro de una celda en la que se hace vacío, y en la medición de la variación de su temperatura absoluta $T$ en función del tiempo $(t)$. Si la desviación de la temperatura del sistema con respecto al estado de equilibrio es pequeña $\left(\Delta T<<T_{0}\right)$, la relajación se manifiesta de acuerdo con la ecuación (1):

$$
T(t)=T_{i} e^{-\frac{t}{\tau}}
$$

Donde $T_{i}$ es la temperatura inicial y $\tau$ es el tiempo de relajación térmica o constante de tiempo del sistema. De este último parámetro se puede obtener la capacidad calorífica por unidad de volumen, $C_{p}$, de la muestra. El calor específico o capacidad de calor específico, $c$, se calcula dividiendo $C_{p}$ entre la densidad, $\rho$ [26].

Mediante el uso de la técnica de RT se determinó la $C_{p}$ de 10 muestras del pegante, elaborado con un procedimiento de síntesis del poliol en el que se aplicó una temperatura de $75{ }^{\circ} \mathrm{C}$ durante 4 horas, mientras se sometió a agitación a $250 \mathrm{rpm}$; la medición de $C_{p}$ se hizo por triplicado. En la figura 9 se muestra el comportamiento típico de las curvas de variación de la temperatura en función del tiempo, correspondiente al calentamiento continuo de una muestra al ser sometida a incidencia de luz láser (línea continua) y al proceso de termalización (línea punteada) cuando ésta se interrumpe.

El valor de $C_{p}$ que se obtuvo a partir de $\tau$ fue de $1.2 \pm 0.1$ $\mathrm{Jcm}^{-3} \mathrm{~K}^{-1}$ y ya que la muestra tiene una densidad promedio de $0.9 \mathrm{gcm}^{-3}$ el valor de $c$ es de $1.1 \pm 0.2 \mathrm{Jg}^{-1} \mathrm{~K}^{-1}$. Este último es igual al reportado para resinas epóxicas de baja conductividad alrededor de $1.1 \mathrm{~J} \mathrm{~g}^{-1} \mathrm{~K}^{-1}$ [27] y de poliuretanos reticulados a base de agua, entre $0.919 \mathrm{y}$ $1.011 \mathrm{~J} \mathrm{~g}^{-1} \mathrm{~K}^{-1}[28]$. 


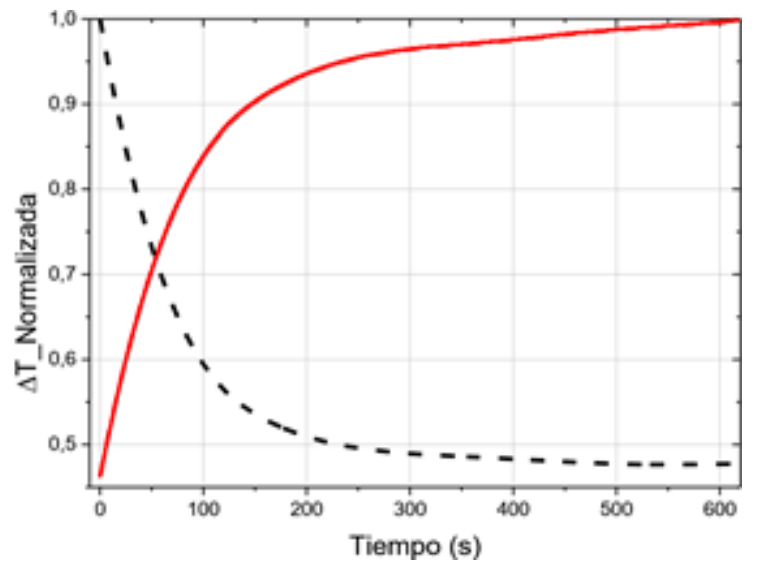

Figura 9. Desviación de la temperatura $(\Delta T)$, normalizada con respecto al valor máximo, en función del tiempo. La medida corresponde a una muestra del pegante (calentamiento línea continua y termalización línea punteada). Fuente: elaboración propia.

\subsubsection{Coeficiente de dilatación (expansión) térmica lineal y difusividad térmica del adhesivo}

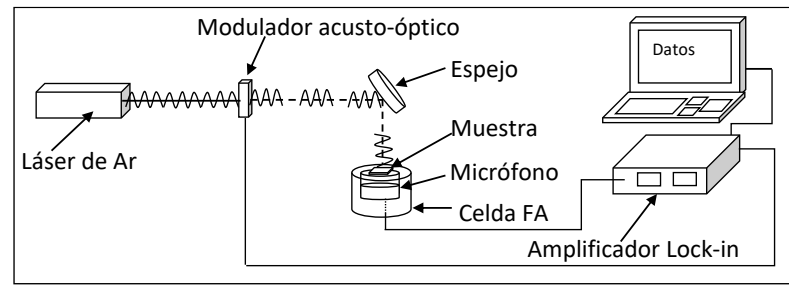

Figura 10. Sistema fotoacústico para la medición de la difusividad térmica y el coeficiente de expansión térmico lineal. Fuente: elaboración propia.

En la figura 10 se muestra un arreglo experimental típico, conocido como configuración en transmisión de calor o resuelta en frecuencia, que mide la amplitud de la señal fotoacústica, $S$, generada en una celda abierta. Este arreglo permite medir de forma directa la difusividad térmica, $\alpha_{s}$, de una muestra sólida en forma de lámina con un espesor definido, así como también su coeficiente de expansión térmico lineal, $\alpha_{T}$. Sobre la celda, la muestra sella herméticamente la cámara de aire del micrófono condensador o de electret. La cara contraria de la muestra se expone a un haz de luz láser (454 nm), modulado en intensidad periódicamente. El calentamiento que se genera en la superficie de la muestra se difunde a través de ésta mediante conducción. Esto genera cambios periódicos de temperatura y por lo tanto de presión, dentro de la cámara de la celda fotoacústica, que son captados por la membrana del micrófono. El amplificador Lock-in extrae la señal de interés y mediante protocolos de comunicación envía a un computador los datos, que luego son tratados con algoritmos de ajuste multiparamétrico.

Empleando esta técnica se midió la difusividad térmica y el coeficiente de expansión térmica lineal del adhesivo natural, lo que conllevó a identificar su intervalo de temperatura de trabajo. Se consideró la respuesta en frecuencia del elemento detector y los posibles mecanismos en la generación de $S$ (difusión térmica, expansión térmica y flexión termoelástica). El método usado en este trabajo, planteado por Bedoya et al [29], es diferente al termomecánico, de tal forma que se perfila en la contribución a la caracterización térmica de adhesivos.

a)

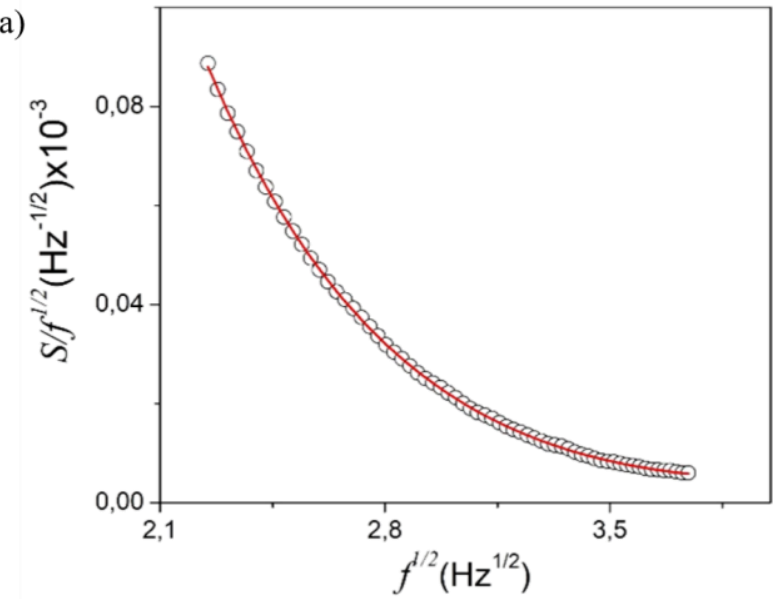

b)

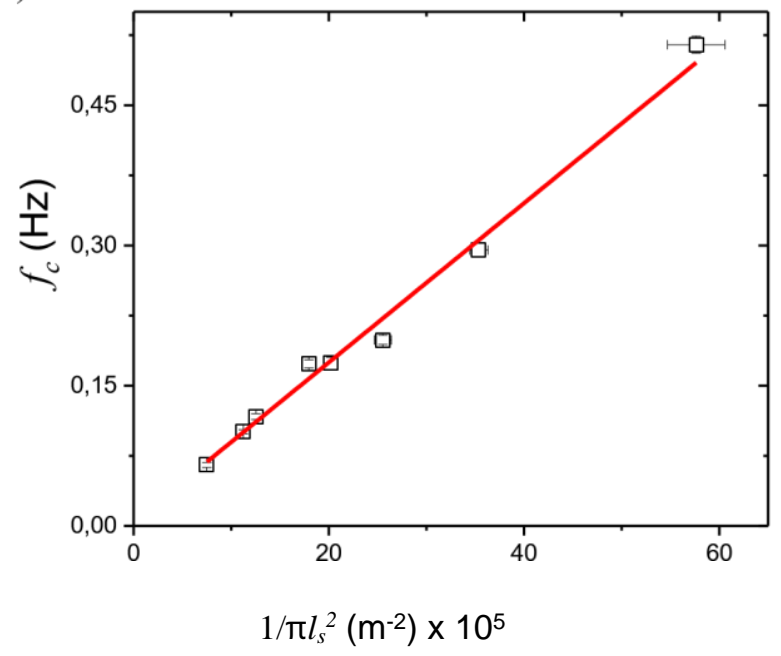

Figura 11. Resultado del ajuste, representado por la línea de color rojo, de a) $S f^{-1 / 2}$ en función de $f^{1 / 2}$ para una muestra de pegante de $235 \mu \mathrm{m}$ de espesor y b) $f_{c}$, de diferentes muestras, en función de $1 / \pi l_{s}^{2}$. Fuente: elaboración propia. 
Inicialmente se obtuvo el valor de la frecuencia de corte, $f_{c}$, aquella para la cual la longitud de difusión térmica es igual al espesor de la muestra, del mejor ajuste de los datos experimentales de la amplitud $S$ divida entre la raíz cuadrada de la frecuencia de modulación del haz de luz incidente, $f$, en función de la raíz cuadrada de $f$, considerando la ecuación 18 de la referencia 29, como se registra en la figura 11 a). Mientras que del ajuste de la curva de la figura $11 \mathrm{~b}$ ), de $f_{c}$ en función de $1 / \pi l_{s}^{2}$, se halló la difusividad térmica, $\alpha_{s}$, teniendo en cuenta que $f_{c}$ $=\alpha_{s} / \pi l_{s}^{2}$ y se obtuvo un valor de $(8.5 \pm 0.4) \times 10^{-8} \mathrm{~m}^{2} \mathrm{~s}^{-1}$.

De acuerdo con el procedimiento propuesto por Bedoya et al, se graficó el valor de $\alpha_{T}$, calculado con la ecuación 19 en la referencia [29], en función de la frecuencia de corte de un conjunto de muestras de adhesivo de diferentes espesores. Los resultados son presentados en la figura 12.

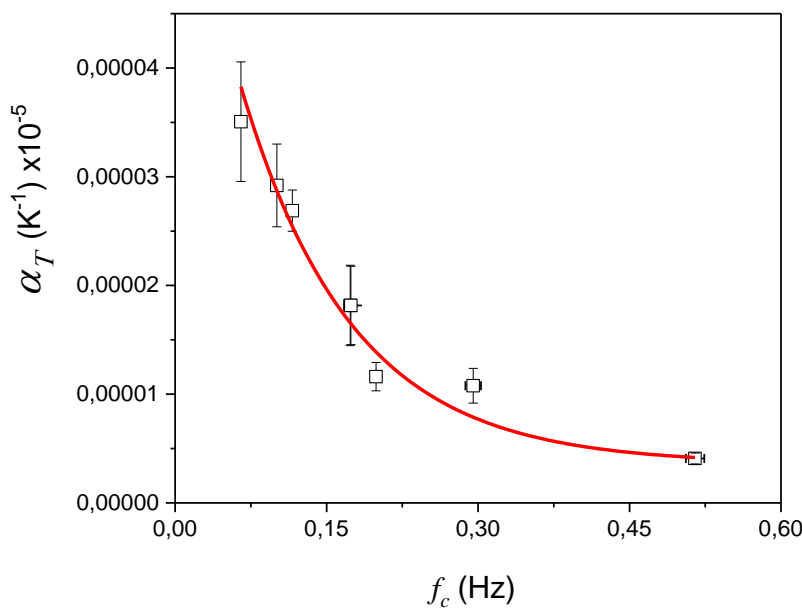

Figura 12. $\alpha_{T}$ dinámico en función de $f_{c}$. La línea roja es la curva de ajuste a una ecuación exponencial decreciente. Fuente: elaboración propia.

El comportamiento de los puntos calculados en la figura 12, se ajustó a una ecuación exponencial: $\alpha_{T}=a_{1} e^{-\frac{f_{c}}{t_{1}}}+$ $a_{2}$. Donde $a_{1}, t_{1}$ y $a_{2}$ son parámetros de ajuste que se obtienen a partir de un método de iteración numérica. La muestra es térmicamente muy gruesa bajo la condición de que $f_{c}$ sea muy pequeño y en esta región el mecanismo de la termoelasticidad predomina sobre la termodifusión $\left(e^{-\frac{f_{c}}{t_{1}}} \approx 1\right)$, de tal forma que se obtuvo un valor $\alpha_{T}=(67 \pm 11) \times 10^{-6} \mathrm{~K}^{-1}$; la incertidumbre de este dato está relacionada con la dificultad para obtener una muestra de espesor uniforme y con la existencia de pequeñas burbujas que se forman en la lámina del pegante luego de secado, lo que es una característica de este material. El signo positivo de este coeficiente implica la expansión del material con el aumento de temperatura y su bajo valor indica que es estable. Este $\alpha_{T}$ es comparable al de Poly(methyl methacrylate) (PMMA), un material transparente termoplástico, que por encima de su $\mathrm{Tg}$ $=105^{\circ} \mathrm{C}$, tiene un coeficiente de $53 \times 10^{-6} \mathrm{~K}^{-1}$ [30], y es mayor que el de la guadua que ha sido estimado en $(27 \pm 7) \times 10^{-6} \mathrm{~K}^{-1}$ [31]; esto se relaciona con la buena flexibilidad de las uniones de los elementos estructurales de guadua.

En la tabla 3 se resumen las características del pegante fabricado con la modificación del aceite vegetal; la efusividad térmica, $\varepsilon$, y la conductividad térmica, $k$, se calcularon con los parámetros medidos, utilizando las relaciones $\varepsilon=\sqrt{k C_{p}}$ y $k=\alpha_{s} C_{p}$ y teniendo en cuenta que la densidad de cada muestra de pegante es aproximadamente $0.9 \mathrm{~g} / \mathrm{cm}^{3}$. El valor estimado de $k$ para el pegante es menor que el de resinas epóxicas, que está entre 0.15 y $0.2 \mathrm{Wm}^{-1} \mathrm{~K}^{-1}$ [20], e incluso inferior al de otros poliuretanos, entre 0.21 y $0.31 \mathrm{Wm}^{-1} \mathrm{~K}^{-1}$; sin embargo, se aproxima más a la de polisacáridos como la celulosa, que está alrededor de $0.071 \mathrm{Wm}^{-1} \mathrm{~K}^{-1}$ [7]. Esta característica revela que el pegante es compatible con la guadua y que tiene características de aislante térmico; lo que se confirma a través del bajo valor de la difusividad térmica, que es del mismo orden que el correspondiente a la guadua, de $11 \pm 1 \times 10^{-8} \mathrm{~m}^{2} \mathrm{~s}^{-1}$ [31].

Tabla 3. Características del polímero con propiedades adhesivas

\begin{tabular}{|l|l|}
\hline $\begin{array}{l}\text { Temperatura de transición } \\
\text { vítrea }\left(\mathbf{T}_{\mathrm{g}}\right)\end{array}$ & $-26.4{ }^{\circ} \mathrm{C}$ \\
\hline Efusividad térmica & $350 \pm 37 \mathrm{Ws}^{1 / 2} \mathrm{~m}^{-2} \mathrm{~K}^{-1}$ \\
\hline Conductividad térmica & $0.10 \pm 0.01 \mathrm{Wm}^{-1} \mathrm{~K}^{-1}$ \\
\hline Difusividad térmica & $8.5 \pm 0.4 \times 10^{-8} \mathrm{~m}^{2} / \mathrm{s}$ \\
\hline $\begin{array}{l}\text { Coeficiente de expansión } \\
\text { térmica }\end{array}$ & $67 \pm 11 \times 10^{-6} \mathrm{~K}^{-1}$ \\
\hline Calor específico & $1.1 \pm 0.2 \mathrm{~J} \mathrm{~g}^{-1} \mathrm{~K}^{-1}$ \\
\hline $\begin{array}{l}\text { Resistencia de compresión } \\
\text { paralela a la fibra }\end{array}$ & $43 \pm 3 \mathrm{MPa}$ \\
\hline $\begin{array}{l}\text { Resistencia cortante paralela a } \\
\text { la fibra }\end{array}$ & $5.9 \pm 0.9 \mathrm{MPa}$ \\
\hline
\end{tabular}

Fuente: elaboración propia.

\subsection{Tablero elaborado con fibra de Guadua Angustifolia Kunth y con el adhesivo}

Con el fin de probar el pegante en la elaboración de aglomerados, se mezcló aserrín de guadua de tamaño de partícula $500 \mu \mathrm{m}$ con el adhesivo de aceite de higuerilla en una relación 1:1, mediante agitación mecánica, aproximadamente por 10 minutos. Este producto se 
depositó en un recipiente cilíndrico y fue prensado con 50 kg-f hasta alcanzar una temperatura de $160{ }^{\circ} \mathrm{C}$, durante 10 minutos y se dejó enfriar a temperatura ambiente; posteriormente, fue separado y secado por 24 horas. El aglomerado en forma de disco, como se muestra en la figura 13, tuvo un diámetro de $11 \mathrm{~cm}$ y espesor 0.72 mm, aproximadamente.

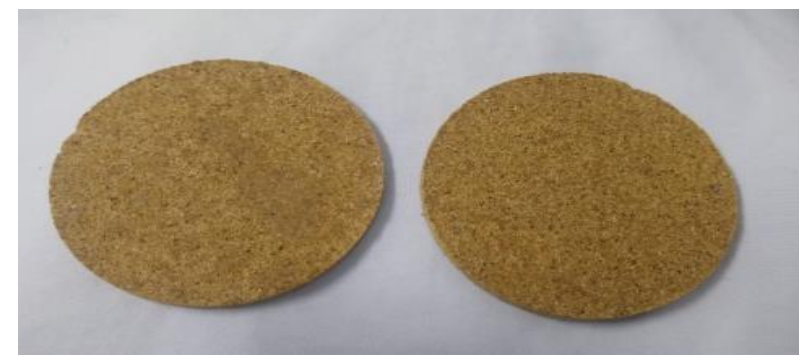

Figura 13. Aglomerado de aserrín de guadua hecho con el adhesivo de aceite de higuerilla. Fuente: elaboración propia.

Se midió el calor específico y la capacidad calorífica por unidad de volumen de una muestra del aglomerado de densidad $0.65 \mathrm{~g} / \mathrm{cm}^{3}$, empleando el método de relajación térmica se obtuvo un valor de $C_{p}=0.83 \pm 0.06 \mathrm{~J} \mathrm{~cm}^{-3} \mathrm{~K}^{-1} \mathrm{y}$ usando la densidad se calculó $c=1.26 \pm 0.03 \mathrm{~J} \mathrm{~g}^{-1} \mathrm{~K}^{-1}$. Este valor de $c$ es similar al reportado para tableros de fibra de densidad media (MDF, sigla en inglés para mediumdensity fibreboard), entre 1.1 y $1.2 \mathrm{~J} \mathrm{~g}^{-1} \mathrm{~K}^{-1}$ para temperaturas entre 40 y $60{ }^{\circ} \mathrm{C}$ [32], y es más alto que el de la fibra de guadua, que está entre 1 y $1.2 \mathrm{~J} \mathrm{~g}^{-1} \mathrm{~K}^{-1}$ para densidades que van de 1,2 a $1,5 \mathrm{~g} / \mathrm{cm}^{3}$ y que el de la fibra de vidrio que está entre 0.796 y $0.81 \mathrm{~J} \mathrm{~g}^{-1} \mathrm{~K}^{-1}$ para densidades entre $2,35-2,5 \mathrm{~g} / \mathrm{cm}^{3}$ [33].

\section{Conclusiones}

Las mediciones indicaron una temperatura de transición vítrea del polímero adhesivo de $-26.4{ }^{\circ} \mathrm{C}$, lo que supera a la de algunos pegantes convencionales. Esto concuerda con el difractograma del material, del que se deduce una composición estructural de cadenas poliméricas entrecruzadas correspondientes a un elastómero flexible y blando.

El adhesivo poliuretano cumple con el $90 \%$ de las características de resistencia a algunos agentes químicos establecidos por la norma ASTM D543-14; su estructura física no se vio afectada por la inmersión en sustancias como agua, etanol, acetona o ácido sulfúrico, con las que se presentaron pérdidas de peso menores al $1 \%$.
De la prueba cortante paralela a la fibra, siguiendo la norma ASTM D143, se obtuvo una resistencia promedio de 5.9 MPa y una resistencia a compresión paralela a la fibra de $43 \mathrm{MPa}$, mientras que la falla de adherencia cohesiva se presentó a $1 \mathrm{MPa}$. Estos valores son comparables a los de pegantes convencionales, reportados por otros autores.

Las mediciones de los parámetros termofísicos del adhesivo permiten especificar que es compatible con la guadua para la elaboración de elementos estructurales, ya que su coeficiente de dilatación térmico es aproximadamente 2.5 veces mayor que el de ésta, lo que permite flexibilidad en las uniones; mientras que los bajos valores de conductividad térmica y de difusividad térmica definen al material como aislante térmico.

El pegante natural se perfila como un producto amigable con el medio ambiente, de potencial aplicación en la industria de los muebles y artesanías con madera y guadua. Considerando la importancia que tienen los ecomateriales, el adhesivo permite la fabricación de aglomerados con el material de desecho de la elaboración de tablillas de guadua (aserrín), con un valor de calor específico comparable al del MDF y con las ventajas de uso de un material que no es derivado del petróleo. Esto no solamente favorecería la sostenibilidad de la producción de elementos estructurales de guadua, sino que también reafirmaría a estos productos dentro del mercado correspondiente

\section{Agradecimientos}

Este trabajo fue financiado por la Universidad del Quindío, a través del proyecto 924. Los autores agradecen el apoyo técnico del personal del Laboratorio de Instrumentación del Programa de Química, del Laboratorio de Estructuras de la Facultad de Ingeniería y del Laboratorio de Fibras Naturales del Instituto Interdisciplinario de las Ciencias de la Universidad del Quindío.

\section{Referencias}

[1] V. A. Pocius, Adhesion and Adhesives Technology. An introduction, 2nd Edition. Ohio, USA: Carl Hanser Verlag, 2002.

[2] R. P. Pohanish, Sittig's Handbook of Toxic and Hazardous Chemicals and cancerigens. Oxford, UK: Elsevier, 2012.

[3] M. Villegas, Bambusa Guadua. Villegas Editores, Bogotá, Colombia, 2005. 
[4] M. F. Valero, “Caracterización Físico-mecánica, Térmica y Morfológica de Polímeros de Redes Interpenetradas con Base en Poliuretano Obtenido a partir de Aceite de Ricino y Almidón Modificados/Polimetilmetacrilato (PMMA)", Polímeros, vol. 21, no. 4, pp. 293-298, 2011, doi: 10.1590/S0104-14282011005000050

[5] M. F. Valero, "Adhesivos Tipo Poliuretano Obtenidos A Partir De Aceite De Ricino Y Almidón Químicamente Modificados", Quim. Nova, vol. 36, no. 5, pp. 657-662, 2013, doi: 10.1590/S010040422013000500008

[6] M. F. Valero, "Determinación de la Densidad de Entrecruzamiento de Poliuretanos Obtenidos a partir de Aceite de Ricino Modificado por Transesterificación", Polímeros: Ciência e Tecnologia, vol. 19, no. 1, pp. 1421, 2009, doi: 10.1590/S0104-14282009000100008

[7] W. Jianye, "Some Mechanical Properties of Typical Polymer-Based Composites", en Physical Properties of Polymers Handbook. Department of Chemistry, Verlag New York, USA: Springer- Verlag, 2007, pp. 487-488.

[8] P.C. Mazo, A. Franco, L. A. Ríos, G. Restrepo, "Obtención de espumas flexibles de poliuretano a partir de aceites de palma y castor modificado", Scientia Et Technica, vol. 1, no. 36, pp. 607-612, 2007, doi: $10.22517 / 23447214.4909$

[9] S. Yano, S. Hirose, H. Hatakeyama, Wood Processing and Utilization. Nueva York, USA, EllisHorwood, 1989.

[10] J. Vega, "Obtención de espumas rígidas de poliuretano a partir de cáscara de piña", Revista Iberoamericana de Polímeros, vol. 9 no.4, pp. 389-407, 2008.

[11] Y. L. Moreno, S. E. Solano, "Utilización del subproducto vegetal generado en la elaboración de edulcorantes de stevia como material de relleno reforzante en elastómeros de poliuretano a partir de aceite de higuerilla", tesis doctoral, Universidad Industrial de Santander, 2008.

[12] J. Vega-Baudrit, M. Sibaja-Ballestero, M. E. Hernández-Hernández, P. Alvarado-Aguilar, "síntesis y caracterización de redes elastoméricas de poliuretano (Epu) utilizado en la elaboración de calzado", Revista Iberoamericana de Polímeros, vol. 7 no. 2, pp. 99-112, 2006.
[13] D. R. Mulinari, C. A. Baptista, G. J. Rocha, A. R. Gonçalves, "Mechanical behaviour of polyurethane from castor oil reinforced sugarcane straw cellulose composites", Procedia Engineering, vol. 10, pp. 20682073, 2011, doi: 10.1016/j.proeng.2011.04.342

[14] I. M. Sulastiningsih, Nurwati, "Physical and mechanical properties of laminated bamboo board", Journal of Tropical Forest Science, vol. 21, no. 3, pp. 246-251, 2009.

[15] H. M. Cleuren, A. B. Henkemans, "Development of the bamboo sector in Ecuador: harnessing the potential of Guadua angustifolia", J Bamboo and Rattan, vol. 2, no. 2, pp. 179-188, 2003.

[16] J. Cardoso, N. Casarotto Filho, P. A. Cauchick, "Application of quality function deployment for the development of an organic product", Food Quality and Preference, vol. 40, pp. 180-190, 2015, doi: 10.1016/j.foodqual.2014.09.012

[17] Department of Agriculture, Forest Service, Forest Products Laboratory, Wood handbook-Wood as an engineering material. Madison, USA: USDA, 2010.

[18] Standard Test Method for Evaluating the Shear Strength of Adhesive Bonds in Laminated Wood Products at Elevated Temperatures, ASTM D724707ae1, 2012.

[19] E. M Petrie, Handbook of Adhesives and Sealants (second Edition). New York, USA: McGraw-Hill, 2007.

[20] S. Ebnesajjad, Handbook of adhesives and Surface Preparation. Technology, Applications and Manufacturing. Introduction and Adhesion Theories, Oxford, UK: Elsevier, 2011.

[21] A. Pizzi, K. L. Mittal, Handbook of adhesive technology. New York, USA: CRC Press, 2017.

[22] U. Henri, Introduction to Industrial Polymers, New York, USA: München Hanser, 1982.

[23] R. Aradhana, S. Mohanty, S. K. Nayak, "Synergistic effect of polypyrrole and reduced graphene oxide on mechanical, electrical and thermal properties of epoxy adhesives", Polymer, vol. 166, pp. 215-228, 2019, doi: 10.1016/j.polymer.2019.02.006

[24] H. J. C. Velásquez, J. A. Osorio-Saraz, J. M. Vélez- Restrepo, "Determinación de la resistencia mecánica a tensión y cizalladura de la Guadua 
angustifolia Kunth", Rev. Fac. Nal. Agr., vol. 58, no. 1, pp. 2709-2715, 2005, doi: 10.15446/rfnam

[25] J. F. Correal, J. S. Echeverry, F. Ramírez, L. E. Yamín, "Experimental evaluation of physical and mechanical properties of Glued Laminated Guadua angustifolia Kunth", Construction and Building Materials, vol. 73, pp. 105-112, 2014, doi: 10.1016/j.conbuildmat.2014.09.056

[26] A. Lara-Bernal, E. Marín, A. Calderón, “Técnica de relajación térmica con excitación variable: caso de la 'rampa' de intensidad', Superficies y vacío, vol. 20, no. 3, pp. 17-20, 2007.

[27] J. McHugh, P. Fideu, A. Herrmann , W. Stark, "Determination and review of specific heat capacity measurements during isothermal cure of an epoxy using TM-DSC and standard DSC techniques", Polymer

Testing, vol. 29, pp. 759-765, 2010, doi: 10.1016/j.polymertesting.2010.04.004

[28] M. H. Kalele, Y. Zhu, M. C. Mulenga, "Preparation and Characterization of Waterborne Polyurethane Crosslinked by Urea Bridges International", Journal of Chemistry, vol. 3, no. 2, pp. 8896, 2011, doi: 10.5539/ijc.v3n2p88

[29] A. Bedoya, E. Marín, A. M. Mansanares, M.A. Zambrano-Arjona, I. Riech, A. Calderón, "On the thermal characterization of solids by photoacoustic calorimetry Thermal diffusivity and linear thermal expansion coefficient", Thermochimica Acta, vol. 614, pp. 52-58, 2015, doi: 10.1016/j.tca.2015.06.009

[30] R. D. Adams, R. D. Adams, J. Comyn, W. C. Wake, Structural Adhesive Joints in Engineering, Netherlands: Springer Science \& Business Media, 1997.

[31] A. Bedoya, F. Gordillo-Delgado, y E. Marín, "Measurement of the thermal expansion coefficient of Guadua angustifolia Kunth using the photoacoustic technique", Journal of Physics: Conference Series, vol. 687 , pp. 1-4, 2016, doi: 10.1088/17426596/687/1/012103

[32] K. Y. Li,n, C.M. Fleischmann, M. J. Spearpoint, "Determining thermal physical properties of pyrolyzing New Zealand medium density fibreboard (MDF)", Chemical Engineering Science, vol. 95, pp. 211-220, 2013, doi: 10.1016/j.ces.2013.03.019

[33] H. P. S. Abdul Khalil, I. U. H. Bhat, M. Jawaid, A. Zaidon, D. Hermawan, Y. S. Hadi, "Bamboo fibre reinforced biocomposites: A review", Materials \& Design, vol. 42, pp. 353-368, 2012, doi: 10.1016/j.matdes.2012.06.015 